\title{
MODELLING OF STIFFNESS VARIABILITY OF SKELETON SLED ON INCLINED ICE PLANE
}

\author{
Martins Irbe, Karlis Agris Gross, Janis Viba, Marina Cerpinska \\ Riga Technical University, Latvia \\ martins.irbe@rtu.lv, kgross@rtu.lv, janis.viba@rtu.lv, marina.cerpinska@rtu.lv
}

\begin{abstract}
This paper continues the previous research for the sliding winter sports taking place on the Bobsleigh and Luge Track. The competition in winter sports is severe. On the ice track of $1200-1500$ meters teams struggle to improve their finish time for one hundredth of a second, and often even for one thousandth of a second. Therefore, on the ice track the slightest improvement of the equipment slideability provides the competitive advantage to the team. The slideability could be improved by the right adjustment of the skeleton sled runner stiffness. This research provides analysis of skeleton sled runner slideability depending on the runner stiffness specifically on the inclined ice plane. To perform the analysis of the motion, first the two dimensional model of the sledge was created. In this model the sledge runner was viewed as on object of 5 elements. The connections of the elements are modelled as joints made of springs to allow small rotational movement. Thus, the model had eight degrees of freedom (DOF) - three DOF for the luge and the driver, and five DOF for the rotation of the joints. For the analysis the physical properties of the connection - elasticity of the spring and damping of the oscillations were discussed. The drag force resulting from the friction with the air affects damping and is in the model included. The modelling results were compared to the site experiments performed on Bobsleigh and Luge Track "Sigulda", Latvia. The track was $23.7 \mathrm{~m}$ long. In the experiments the stiffness of the runners was subject of variability. In addition, the temperature of the air, humidity of the air and the temperature of the ice on the track were measured. The results of the experiments and modelling revealed that there was a non-linear relationship between the change of the runner stiffness and velocity. The wavy shapes depend on the characteristic of material elasticity. Also the sliding friction frequency depends on the stiffness and runners profile.
\end{abstract}

Keywords: winter sports, skeleton, sliding motion, modelling, runner stiffness.

\section{Introduction}

Previous studies looked at the movement of the skeleton sleigh on inclined harmonious function profile of roughness with variable runner stiffness [1]. How the movement is affected by the resistance forces - the air resistance $C_{D}$ and the ice friction coefficient $f$-was analyzed. The analysis used a mathematical model calibrated with experimental acceleration measurement data from the ice track.

Working on the effect of changes in the stiffness of the skeleton runners on slideability continues. Special attention is paid to cooperation with the athletes of the Latvian skeleton Olympic team. In consultations with the athletes, the nuances are clarified and the research direction adjusted to get the most practical input. The most important thing for athletes is to slide through the track in the shortest time possible.

Skeleton is the only sliding sport where runner stiffness is regulated. For athletes, the softer or less sting stiffness is more comfortable, because it ensures easier handling; however, it slows down the speed of the sliding. The purpose of this study is to find out, if, when increasing the runner stiffness, the sliding speed increases unlimitedly in the range used by beginners and professional athletes; to determine whether there is an optimum point and what factors are more likely to influence it.

In order to achieve the goal of the study, a 2 dimensional skeleton and slider model has been developed, where the change of runner stiffness is analyzed by dividing the runner into 6 elements and connecting those with five rotational spring pins, which spring elasticity and damping parameters can be changed. Unlike the previous study with a two-degree of freedom model, the 8-degree of freedom model is used here. The simulation results are compared with practical experiments from the start-up platform of the Bobsleigh and Luge Track in Sigulda.

The start of the skeleton event has been analysed from the moment, at which the athlete commences running until lying down onto the skeleton [2-4]. More about bobsled contact force components on ice track curves is expressed in [5], the sum of contact forces on the runners divided by the bobsled mass compared with accelerations measurements. A good agreement can be generally noticed between the force and acceleration measurements, especially for the vertical direction. Which means that it can be predicted what could be the contact forces. 
The vibrations and the ways they can influence the friction between the ski running surface and the snow is described in [6;7]. The papers describe the results of experimental studies on the selfinduced and forced vibrations of loaded cross-country skis. However, the vibration of the metal runners has not been studied before in detail.

\section{Runner stiffness modelling with WorkingModel 2D}

The task of modeling was to find out the effect of two symmetrical runner's elasticity (stiffness) changes on the skeleton's slideability. A skeleton sliding motion model in a 2-dimensional plane $(X$, $Y$ ) was created (Fig. 1).

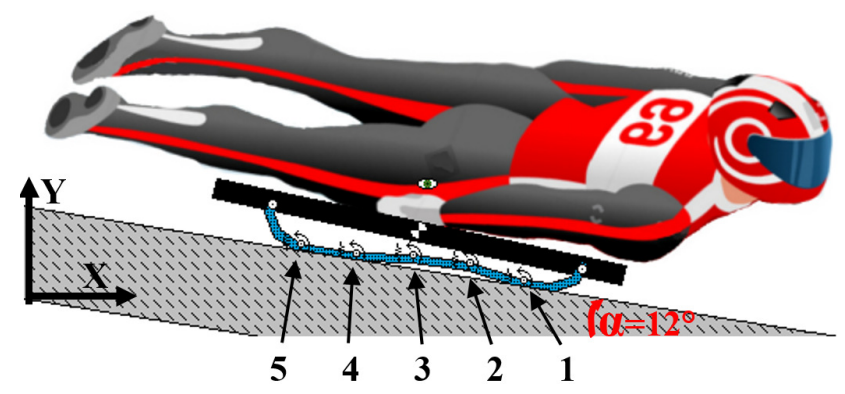

Fig. 1. Skeleton and slider 2D model: with five rotational spring pins

Skeleton's runner is divided into 6 elements, which are connected with identical rotational spring pins, which elasticity (stiffness) parameters can be changed. The model has a total of 8 degrees of freedom: three levels of freedom for the sledge along with a slider and another 5 degrees of freedom for rotational spring pins. A Runge-Kutta-Merson (RKM) numerical integration solver built-in WorkingModel is used for the sliding model analysis with integration time step $0.001 \mathrm{~s}$.

For the created model motion modeling was performed at the system parameters, such as: $m_{1}=65 \mathrm{~kg}-$ wright of the slider; $m_{2}=32 \mathrm{~kg}$ - weight of the skeleton; $\alpha=12^{\circ}-$ inclination angle of the track; $f=0.03$ - ice and runner interaction slip friction coefficient; $b_{0}=0.4$ - air resistance coefficient of the skeleton mass center motion on the slope; $\beta=2^{\circ}$ - angle between rotational spring pins. $k$ - spring elasticity (stiffness) parameter; $b$ - spring damping coefficient. The effect of the last two parameter value changes on the skeleton model sliding distance modeling up to 5 seconds is shown in Figures 2 and 3.

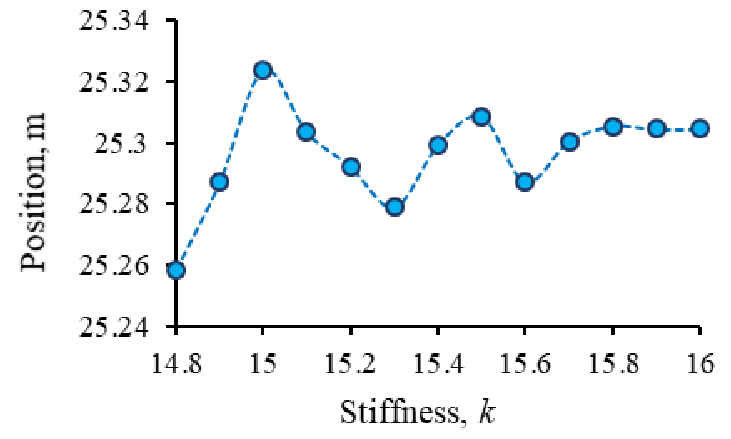

Fig. 2. Sliding position after 5 sec. with different stiffness coefficient $k$

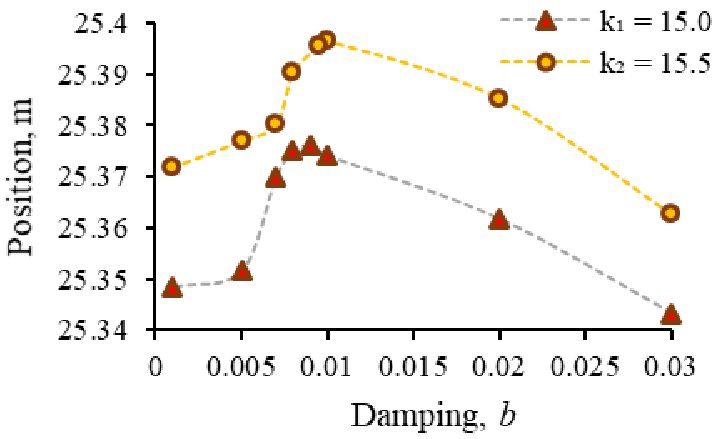

Fig. 3. Sliding position after 5 sec. with different damping coefficient $b$

The largest displacement of the skeleton sleigh in the respective range is performed at the stiffness coefficient $k_{1}=15$ and $k_{2}=15.5$ when $b=0$ (Fig. 2). In the graph, Figure 3, there is a comparison of how the skeleton's slideability is influenced by the second physical characteristic element of the spring - damping coefficient $b$ (when $k_{1}=15$ and $k_{2}=15.5$ ). There is a clearly visible optimum point at which the sleigh slides better. It can be seen that the spring stiffness damping improves the slip at $k_{1}=15$ and $b_{1}=0.009$, when the traveled road increases by $5.2 \mathrm{~cm}(0.2 \%)$, while with $k_{2}=15.5$ and $b_{2}=0.01$ it is driven by $8.7 \mathrm{~cm}(0.34 \%)$ more than without damping. More significant damping improvements are with higher $k$-values, and the value of $b$ should also be increased when increasing $k$. 
In the following Figure 4, the directions of action of the forces in the rotational spring pins are shown and their values at the time $t=4.7 \mathrm{~s}$, at the speed $v=9.08 \mathrm{~m} \cdot \mathrm{s}^{-1}$. Acceleration is parallel to the sliding plane with a value of $\sim 1.8 \mathrm{~m} \cdot \mathrm{s}^{-2}$. Forces 2,3 and 4 in the rotational pin are parallel to the contact surface and the values of their modules are lower than in the pins 1 and 5, which, in addition to the compressive force, are also exposed to the bending moment.

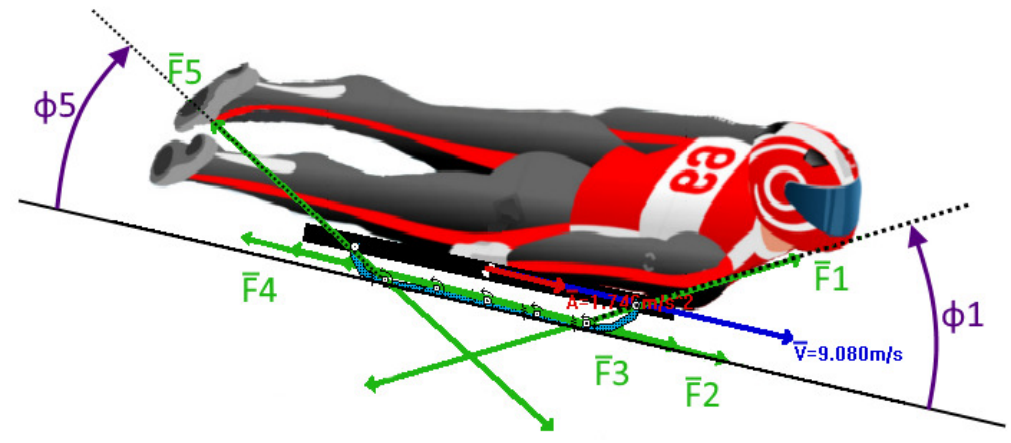

Fig. 4. Skeleton and slider 2D model: with velocity, acceleration and force vector directions

As the spring stiffness increases, the force values increase accordingly, but their direction of action does not change significantly in the pins 2, 3 and 4.But in the pins 1 and 5 the angles $\varphi_{1}$ and $\varphi_{5}$ of the direction of the force change, where the angle decreases as the stiffness of the runner increases.

\section{Experiment of the skeleton on the ice track}

An experiment was conducted on the ice track to determine the similarity in the outcome to the numerical model and to verify the stiffness of the sliding motion, using a skeleton sledge that meets the requirements of the IBSF [8]. The experiment was conducted on a straight $23.7 \mathrm{~m}$ long ice track angled at $\alpha=12^{\circ}$. Figure 5 shows the location of the accelerometer fixed to the underside of the skeleton at the centre of mass. The reference position $r=0 \mathrm{~mm}$, the runner is completely nontensioned $F=0 \mathrm{~N}$. Athlete tensioning of the runners is done depending on the degree of mastery, for beginners $r=6 \mathrm{~mm}$, however, for professional athletes 10 to $12 \mathrm{~mm}$.

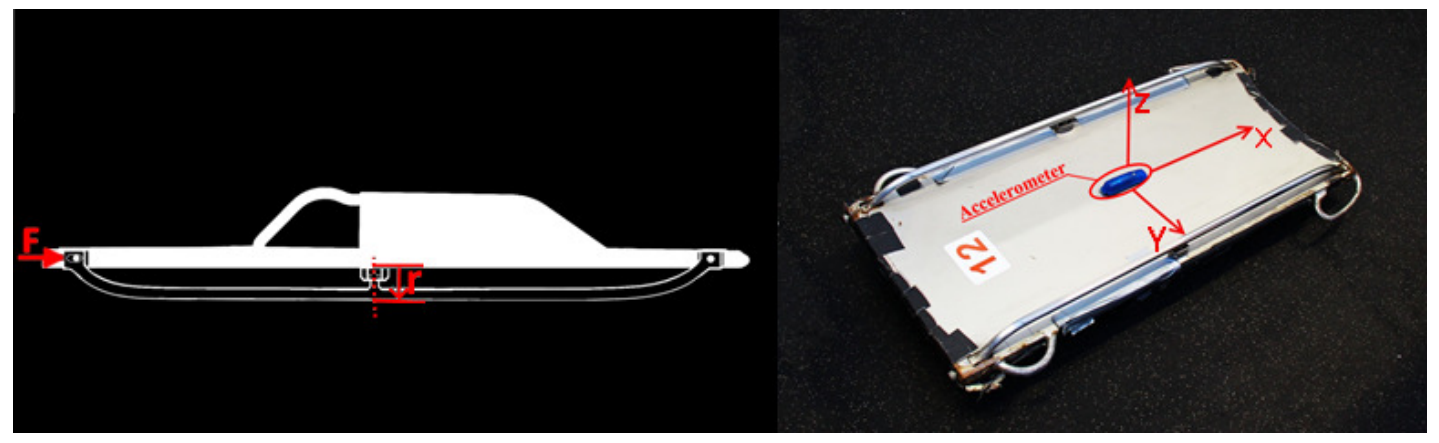

Fig. 5. Runner tension schematics and skeleton sled equipped with accelerometer and measuring axis directions

The sliding time is measured with optical sensors at the top and bottom of the incline after the skeleton starts sliding from a stationary position. This sliding time was used to calibrate the accelerometer data. For motion analysis, data from the 3-speed portable Accelerometer X16-1D are used, which is fixed to the lower part of the sled on the plane surface and is oriented towards axial directions, Figure 5, right. The data recording frequency is $400 \mathrm{~Hz}$.

\section{Results and discussion}

The parameters characterizing the environment of the day of the experiments are summarized in Figure 6 . The biggest changes in impact are at the last rides $r=11.5 \mathrm{~mm}$ and $12 \mathrm{~mm}$ runner stiffness. Since the experiment takes several hours, environmental changes are inevitable.

Figure 7 compares the skeleton sliding time, which is measured with the track optical sensor time measurement device (accurate to $0.001 \mathrm{~s}$ ), and the average value of the acceleration $a_{x}$, measured with 
a portable accelerometer. The line of linear approximation of data points to the coherence of data - as the time spent on the track decreases, the average value of the acceleration increases. Only the acceleration values of one measurement series at $r=11.5 \mathrm{~mm}$ have a relatively large offset, possibly associated with the measurement error in the experiment. It can be seen that the average value of $a_{x}$ in both the experiment and 2D model is $\sim 1.8 \mathrm{~m} \cdot \mathrm{s}^{-2}$ and depends on the ice friction coefficient (in the experimental results acceleration values are given without $g=9.81 \mathrm{~m} \cdot \mathrm{s}^{-2}$ free fall acceleration).

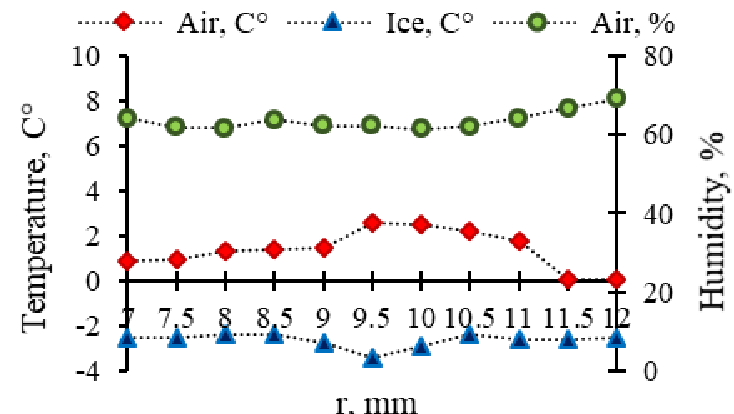

Fig. 6. Air and ice temperature and air humidity changes affecting runner's stiffness

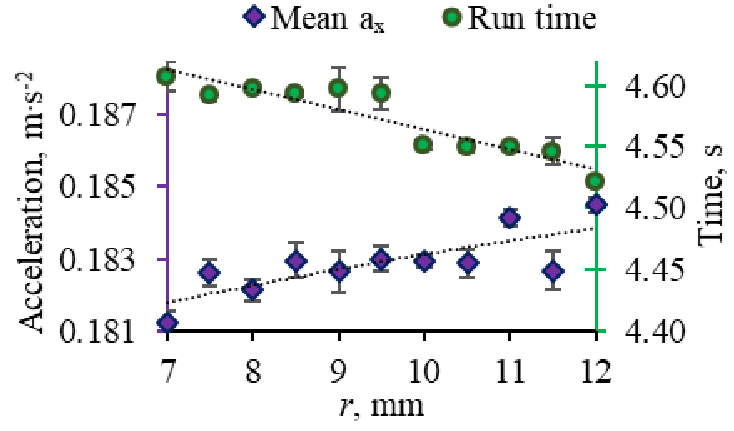

Fig. 7. Sliding direction $\mathrm{X}$-axis accelerations and time measurements depending on runner's stiffness

For further analysis, the $X$-axis of the direction of motion and the perpendicular to the base $Z$-axis acceleration values are considered. Transverse accelerations are not considered, because this movement is limited by the ice groove, on which the runner slides. The following Figure 8 shows the average acceleration amplitudes, where the $a_{x}$ value starts to rise rapidly behind the $r=10 \mathrm{~mm}$ runner stiffness and decreases again at $r=12 \mathrm{~mm}$. Accelerations from contact with the base $a_{z}$ vary in wavelengths and are bigger than $a_{x}$, and reach the maximum at $r=11.5 \mathrm{~mm}$.

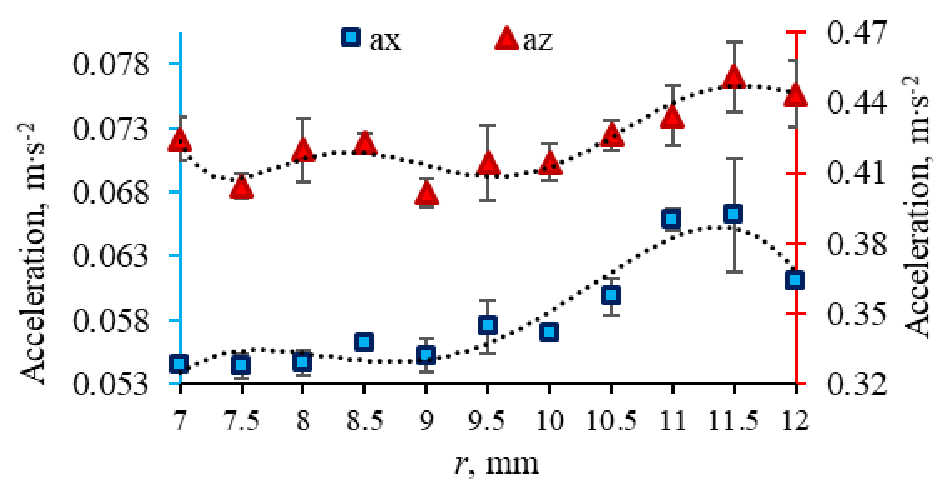

Fig. 8. Mean acceleration amplitude in $X$ and $Z$ axis direction depending on runner's stiffness

The acceleration data signal is processed using FFT (Fast Fourier Transform). Figures 9 and 10 show the $a_{x}$ and $a_{z}$ spectral graphs respectively (for the above mentioned extreme case $r=11.5 \mathrm{~mm}$ ). Movement acceleration has one characteristic frequency around $5-7 \mathrm{~Hz}$, while Z-axis accelerations have two: first $7-8 \mathrm{~Hz}$ and second at $\sim 70 \mathrm{~Hz}$. In this study, only the first characteristic frequencies that characterize the skeleton sleigh movement and the friction process between the runner and the ice are considered.

The characteristic frequencies and peak heights of both axes are summarized in the following Figures 11 and 12. The dotted line for $a_{x}$ and $a_{z}$ peak heights is described by the 3rd polynomial of the $X$-axis and the 4th polynomial of the Z-axis. The characteristic frequencies in Figure 12 also change nonlinearly, the $Z$-axis direction frequencies can be described by the 3rd order polynomial. On the other hand, much higher frequency in the $X$-axis is at low stiffness with a tendency to decrease with the stiffness increasing. 


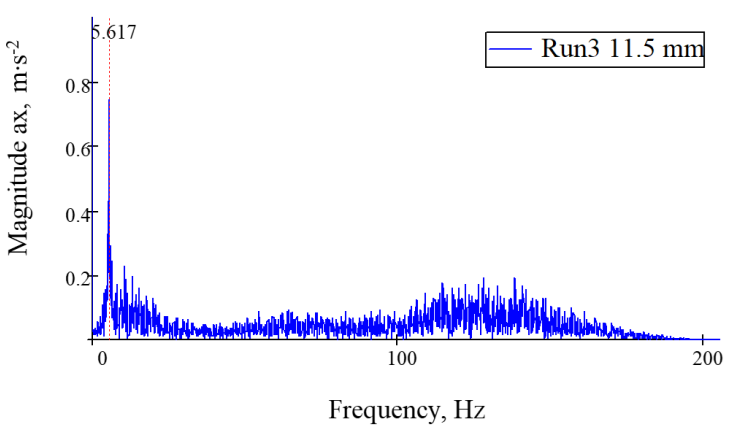

Fig. 9. Accelerated data signal spectrum $a_{x}$

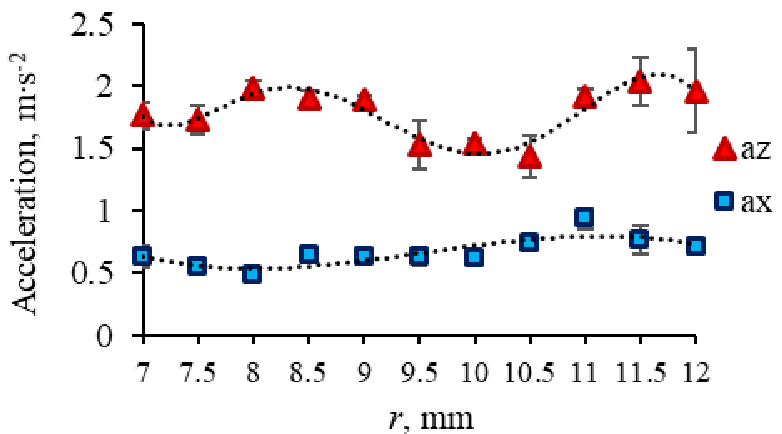

Fig. 11. Magnitude of acceleration depending on runner's stiffness

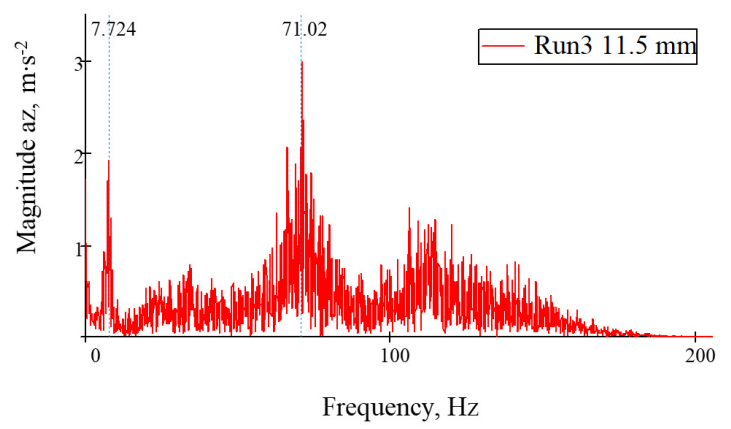

Fig. 10. Accelerated data signal spectrum $a_{z}$

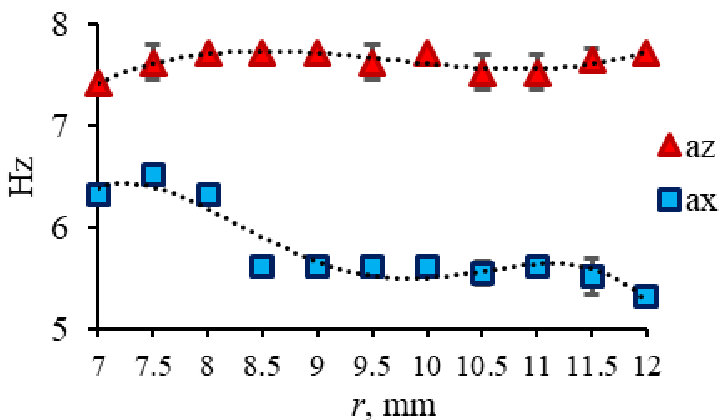

Fig. 12. Characteristic frequency depending on runner's stiffness

Similarly, as when modelling with a 2D model in Figures 2 and 3, the physical experiments also demonstrate the effect of non-linearity of the skeleton runner on the result.

\section{Conclusions}

1. The stiffness of the skeleton sleigh influences the direction of the forces $\varphi_{1}$ and $\varphi_{5}$, where the angle decreases as the stiffness of the runner increases and the contact force is distributed more evenly through the runner profile.

2. Increasing the stiffness of the skeleton runners, the friction frequency decreases for acceleration oscillation in the sliding direction and increases in the vertical direction.

3. From the physical experiments and 2D modelling it can be concluded that the effect of the runner stiffness is highly nonlinear and of wavy shapes, and depends on the characteristic of material elasticity.

\section{Acknowledgements}

This work has been supported by the ERDF project "The quest for disclosing how surface characteristics affect slideability" (No.1.1.1.1/16/A/129).

The authors gratefully acknowledge the assistance from Dainis Dukurs, Inga Reininga, Ernests Jansons and Liene Pluduma for assistance with the experiments at the bobsled push-start facility.

\section{References}

[1] Irbe M., Gross K. A., Viba J., Cerpinsks M. Analysis of the acceleration and numerical modeling of the skeleton sled motion, Proceedings of International conference "Eng. Rural Dev. 17", 2018, pp. 1401-1406.

[2] Bullock N., Martin D., Ross A., Rosemond D., Holland T. and Marino F. Characteristics of the start in women's World Cup skeleton Sport. Biomech 7, 2008, pp. 351-60.

[3] Roberts I. J. M. Skeleton bobsleigh mechanics: athlete-sled interaction. Ph.D. thesis, The University of Edinburgh, 2013. 
[4] Zanoletti C. et al., Relationship between push phase and final race time in skeleton performance. J. Strength Condit. Res. 20(3), 2006, pp. 570-583.

[5] Braghin F. et al., Experimental assessment of Bobsleigh dynamics and ice-skate contact forces. Top. Modal Anal. II 6, 2012, pp. 487-498.

[6] Koptyug A. et al. Cross-country ski vibrations and possible mechanisms of their influence on the free gliding. Procedia Eng. 34, 2012, pp. 473-478.

[7] Koptyug A., Kuzmin L.,Experimental field studies of the cross-country ski running surface interaction with snow. Procedia Eng. 13, 2011, pp. 23-29.

[8] IBSF 2015 International Skeleton Rules IBSF 56. 